\title{
Integrating Electrokinetic and Bioremediation Process for Treating Oil Contaminated Low Permeability Soil
}

\author{
Bimastyaji Surya Ramadan ${ }^{1 *}$, Agus Jatnika Effendi², and Qomarudin Helmy ${ }^{2}$ \\ ${ }^{1}$ Master Program of Environmental Engineering, Environmental Engineering Department, Bandung Institute of Technology, Indonesia \\ ${ }^{2}$ Environmental Engineering Department, Bandung Institute of Technology, Indonesia
}

\begin{abstract}
Traditional oil mining activities always ignores environmental regulation which may cause contamination in soil and environment. Crude oil contamination in low-permeability soil complicates recovery process because it requires substantial energy for excavating and crushing the soil. Electrokinetic technology can be used as an alternative technology to treat contaminated soil and improve bioremediation process (biostimulation) through transfer of ions and nutrient that support microorganism growth. This study was conducted using a combination of electrokinetic and bioremediation processes. Result shows that the application of electrokinetic and bioremediation in low permeability soils can provide hydrocarbon removal efficiency up to $46,3 \%$ in 7 days operation. The highest amount of microorganism can be found in 3-days operation, which is $2 \times 10^{8} \mathrm{CFU} / \mathrm{ml}$ using surfactant as flushing fluid for solubilizing hydrocarbon molecules. Enhancing bioremediation using electrokinetic process is very potential to recover oil contaminated low permeability soil in the future.
\end{abstract}

\section{Introduction}

Traditional oil mining activities in Indonesia is increasing every year especially in places with high crude oil reserves. Oil resources are non-renewable energy resource that is being a primary fuel for society and industries. The development of this industry certainly has an impact, either directly or indirectly. Economic, environmental, and socio-cultural impacts can be either positive or negative. Counter and recovery measures from the negative impacts that will occur should be done so it will not harm the environment [1].

Crude oil is a combination of various hydrocarbon chemical compounds from short to long chains that are difficult to mineralize. Various activities of oil mining industry such as exploration, exploitation, extraction, processing, transportation and marketing of processed oil products potentially produce waste (byproduct) that can pollute the environment. Oil contains aliphatic and aromatic compound and some hazardous materials such as benzene, ethyl benzene, toluene, and xylene (BTEX) that can accumulate in human bodies and living things, destroy the ecosystems, and pollute the soil and groundwater environment [2]. Indonesian government has been already concerned about soil contaminated with petroleum hydrocarbon as stated in Ministerial Decree of Environment Number 128/2003 about Procedures and Technical Requirements for Processing Waste Oil and Contaminated Soil. In that regulation, petrochemical compound in contaminated soil is measured as Total Petroleum Hydrocarbon (TPH).
Various kinds of remediation strategies due to crude oil pollution have been widely studied from physics, chemistry, and biology methods. Remediation of contaminated soil using chemical-physics methods such as solidification, stabilization, thermal, soil washing, gas and liquid extraction or a combination of various technologies is usually expensive, and can produce hazardous compound [3]. Therefore, bioremediation then used as a alternative technology that attract great attention from practitioner because it can eliminate pollutants easily through biodegradation process at low invesment cost. Nevertheless, the process of bioremediation requires long operational time so that there is a need for finding a right strategy to improve the process efficiency [4].

Electrokinetic technology is known to be used as a alternative for treating contaminated soil using constant low voltage gradient [5], [6]. The electrochemical process is used to improve bioavailability and biomixing on contaminated soil so that improving the efficiency and effectiveness of the bioremediation process. The weakness of this technology is the availability of electrical energy resources to supply the needs of electric current. In addition, removal efficiency of contaminant is strongly influenced by the complexity of the soil environment both in terms of chemical, physical and biological. Drastic changes in $\mathrm{pH}$ and high transport velocity of soil compounds or ions due to electrochemical process can negatively affect the soil environment to be toxic to microorganisms. This technology has a good removal efficiency on clay soil that have low hydraulic permeability [7]. 
Implementation of electrical current can directly affect the soil condition chemically and biologically so that the need for research that discusses the interaction between the application of electrokinetic remediation technology by indigenous microorganism. The combination of electrokinetic and bioremediation technologies is also known to have greater efficiency when compared to the application of individual technologies [8]. Therefore, a laboratory scale study is needed as a guide for the application of this technology in Indonesia.

In this area of research, there are 4 variations that we compared: bioremediation (bio), electrokinetic bioremediation without any development (EK-Bio), electrokinetic bioremediation with polarity reversal (PREK-Bio) and electrokinetic bioremediation with addition of tween 80 surfactant (EKSF-Bio). The study was conducted on a laboratory scale by taking the contaminated soil from around traditional oil mining in Grobogan District, Central Java, Indonesia.

\section{Methods}

\subsection{Lab Scale Set-up}

The reactor set up that has been used in this study can be seen in Figure 1. The reactor is made of acrylic glass which is divided into 3 main chamber: overflow collector chamber, electrolyte chamber, and soil chamber. The dimension of soil chamber is $18 \times 12 \times 10 \mathrm{~cm}^{3}$. Contaminated soil is located in the center of the reactor and flanked by an electrolyte chamber containing carbon electrodes with dimensions of $9 \times 9 \times 0.5 \mathrm{~cm}^{3}$. The carbon electrode is connected to a power supply device (Sanfix, DC Power supply) with a fixed voltage of $0.5 \mathrm{~V} / \mathrm{cm}$. Both electrolyte chambers are connected with an collector chamber to accommodate overflow of water due to electroosmosis and electrolysis process. Electrolyte and soil chamber is separated by whatmann filter paper and porous acrylic glass with a pore diameter of $0.3 \mathrm{~cm}$ and $0.5 \mathrm{~cm}$ in space for each pore.

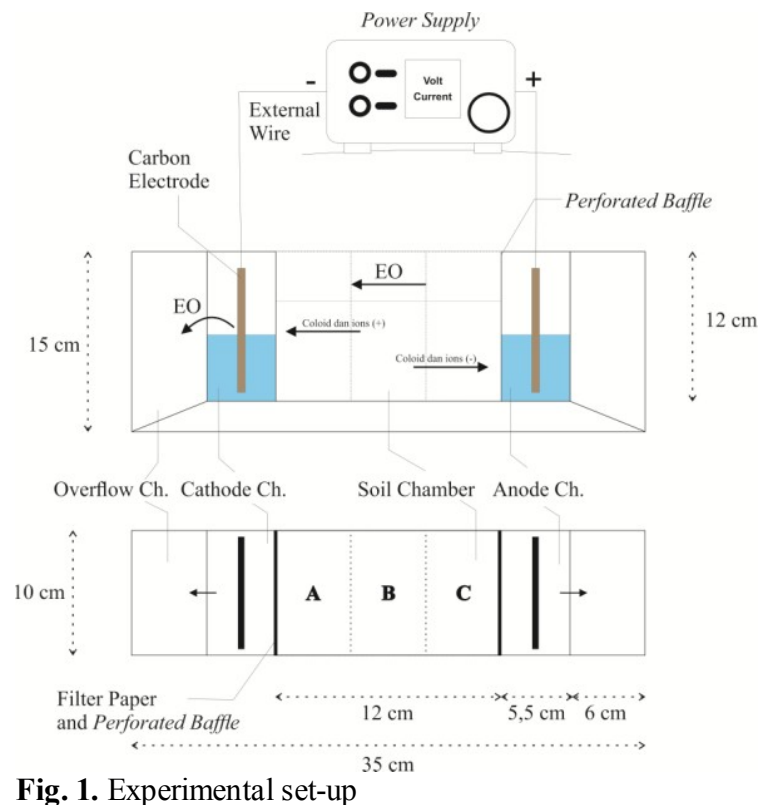

\subsection{Materials}

\subsubsection{Soil}

Oil-contaminated low permeability soil is derived from Bandarharjo village, Grobogan, Central Java around tradtional oil drilling well which managed independently by the local community. Physical tests of soil parameter were conducted in Soil Mechanics Laboratory of Civil Engineering ITB while soil chemical properties were carried out at the Center for Research and Development of Mineral and Coal Technology, Bandung. Microbiology testing was conducted in Environmental Microbiology Laboratory of ITB. From the XRD test results, it is known that the soil contains quartz, montmorillonite, kaolinite and calcite mineral. Detailed soil characteristics can be seen in table 1.

Prior to use in the study, soils that had been taken from the field were prepared first. The soil is air-dried and pounded using mortar. Then, soil is sieved in $2 \mathrm{~mm}$ pore sieve to remove rack, twigs and grass. In order to increase homogeneity, soil is mixed using cone and quartering methods. An electrokinetic reactor requires 1,500 grams of dry soil.

Table 1. Soil properties

\begin{tabular}{llll}
\hline Parameter & & Parameter & \\
\hline Gravel (\%) & 5,2 & Cation Exchange & 30,48 \\
Sand (\%) & 15,81 & Capacity (meq/100g) & \\
Silt (\%) & 45,46 & Organic Carbon (\%) & 5,07 \\
Clay (\%) & 33,53 & N Total (\%) & 0,137 \\
Hydraulic & 0,014 & P Total (mg/kg) & 329 \\
Conductivity & $9,6 \times 10^{-7}$ & C/N Ratio & 37,08 \\
Permeability & & Electrical & 243 \\
Plasticity Index & 111 & Conductivity & \\
pH & $5,5-6$ & $(\mu \mathrm{S} / \mathrm{cm})$ & \\
\hline
\end{tabular}

\subsubsection{Micro-organisms}

The microorganisms consortium was obtained from the original soil around traditional oil well and propagated in SBS solution with medium composition per $500 \mathrm{ml}$ of aquadest was $0.45 \mathrm{~g} \mathrm{KH}_{2} \mathrm{PO}_{4} ; 0.135 \mathrm{~g} \mathrm{~K}_{2} \mathrm{HPO}_{4} ; 0.135 \mathrm{~g}$ $\left(\mathrm{NH}_{4}\right) 2 \mathrm{SO}_{4} ; 0.06 \mathrm{MgSO}_{4}$; And 0.135 yeast extract. The microorganism propagation was then continued using aquadest with dextrose substrate for 2 months until TSS reached $\pm 2.000 \mathrm{mg} / 1$ of concentration.

The ideal water content for bioremediation process is $30-60 \%$. In this study, aquadest was added to dry soil of $750 \mathrm{ml}$ and $1 \%$ of microorganism suspension. The soil, water and microbial suspension are then mixed manually and placed in an electrochemical reactor.

\subsubsection{Hydrocarbons}

In this study, crude oil is analyzed quantitatively and qualitatively. The concentration of oil was analyzed quantitatively in Total Petroleum Hydrocarbon units and qualitative using Gas Chromatography Mass Spectroscopy (GC-MS) method. The soxhlet extraction method is used to extract oil from the soil in accordance 
with EPA 9071B on n-hexane extractable material (hem) for sludge, sediment, and solid samples. The carbon compound contained in the oil extraction can be seen in Table 2. The concentration of TPH at the beginning of the study was $3.3509 \%$.

Table 2. Hydrocarbon compound from soil extraction

\begin{tabular}{l|lll}
\hline No & Compound Name & MW & Formula \\
\hline 1 & $\begin{array}{l}\text { Alpha-Muurolane \$\$ } \\
\text { Naphthalene, decahydro-1,6- } \\
\text { dimethyl-4-(1-methylethyl)- }\end{array}$ & 208 & $\mathrm{C}_{15} \mathrm{H}_{28}$ \\
2 & $\begin{array}{l}\text { 4,5-alpha,alpha-Eudesmane \$\$ } \\
\text { 4a,8-dimethyl-2-isopropyl } \\
\text { perhydronaphthalene }\end{array}$ & 208 & $\mathrm{C}_{15} \mathrm{H}_{28}$ \\
3 & $\begin{array}{l}\text { Phytane \$ Hexadecane, } \\
\text { 2,6,10,14-tetramethyl- }\end{array}$ & 282 & $\mathrm{C}_{20} \mathrm{H}_{42}$ \\
4 & $\begin{array}{l}\text { Hexadecane } \\
\text { Cadalene \$\$ Naphthalene, 1,6- } \\
\text { dimethyl-4-(1-methylethyl) }\end{array}$ & 226 & $\mathrm{C}_{16} \mathrm{H}_{34}$ \\
6 & $\begin{array}{l}\text { Norphytane \$\$ Pentadecane, } \\
\text { 2,6,10,14-tetramethyl }\end{array}$ & 268 & $\mathrm{C}_{15} \mathrm{H}_{18}$ \\
7 & $\begin{array}{l}\text { Eicosane } \\
{ }_{19} \mathrm{H}_{40}\end{array}$ \\
8 & Heneicosane & 282 & $\mathrm{C}_{20} \mathrm{H}_{42}$ \\
\hline
\end{tabular}

\subsection{Experimental Procedure}

This study was conducted by varying four batch studies conducted at room temperature which applying a constant voltage of $18 \mathrm{~V}$ in three reactor and without applying an electric field in the last reactor. The first test uses bioattenuation placed on the reactor in the absence of an electrokinetic treatment (Bio). The second test is an electrokinetic bioremediation test without reversing the polarity during running time by using aquadest as its electrolyte solution (EK-Bio). The third test is in the same principle as the second test but uses tween 80 as a flushing fluid as well as an electrolyte (EKSF-Bio). The fourth test uses aquadest as electrolyte and polarity reversal (PR-EK-Bio). The surfactant used in this study is a biodegradable tween 80 surfactant which has a low $\mathrm{CMC}$ value. The concentration of surfactant used was 1 $\mathrm{g} / \mathrm{L}$. The research duration is 7 days which is intended to see the efficiency of TPH removal in a short period of time. In summary, the conclusions of the four tests that have been done can be seen in Table 3 .

Table 3. Summary of EK Test

\begin{tabular}{l|ll}
\hline Test & Explanation & Electrolyte \\
\hline Bio & Bioremediation & No \\
EK-Bio & Electrokinetic & Aquadest \\
& Bioremediation & \\
EKSF-Bio & $\begin{array}{l}\text { Electrokinetic Soil } \\
\text { Flushing }\end{array}$ & Tween 80 \\
& $\begin{array}{l}\text { Bioremediation } \\
\text { PR-EK-Bio }\end{array}$ & $\begin{array}{l}\text { Polarity Reversal } \\
\text { Electrokinetic }\end{array}$ \\
& Aquadest \\
& Bioremediation & \\
\hline
\end{tabular}

\subsection{Sampling and Analysis}

The main parameters to be analyzed are the number of microorganisms represented in total plate count (TPC) and total petroleum hydrocarbon (TPH). Both parameters are taken on a daily basis of $0,1,2,3$ and $7^{\text {th }}$ day of the study. Other parameters that may affect processes such as temperature, $\mathrm{pH}$, and moisture are tested daily. Multimeters are used to ascertain whether the voltage generated by each power supply is the same and constant every day. Detailed sampling location can be seen in Figure 2. The entire sample was tested in triplicate.

Temperature and $\mathrm{pH}$ were tested using a portable sampler available in the laboratory. Water content was analyzed by gravimetry by taking 30-40 g samples and air-dried it then weighed again. The difference between wet and dry weight is the moisture content of the sample. The TPH analysis is performed by soxhlet extraction as described in point 2.2.2. TPC was analyzed using pour plate method on NA medium with composition: $1 \mathrm{~g}$ beef extract, $2 \mathrm{~g}$ yeast extract, $5 \mathrm{~g}$ pepton, $5 \mathrm{~g} \mathrm{NaCl}$, and $15 \mathrm{~g}$ agar and mixed it in 1 liter of distilled water.

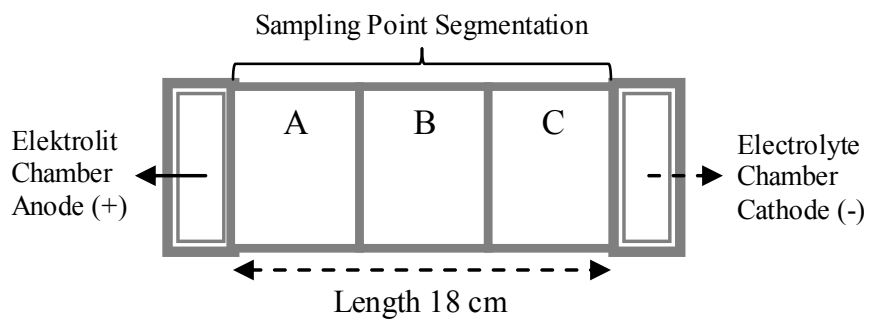

Fig. 2. Sampling point

\section{Results and Discussion}

\subsection{Effect on Soil Condition}

Water content is an important factor for microorganisms activity in the soil. Water can stimulate microorganisms to perform metabolism. Figure 3 shows that in the Bio test, no electroosmosis process occurs so that the water in the soil evaporates over time. The existence of electroosmosis process allows the movement of water from electrolyte chamber into the pores of the soil. In the EK-Bio and EKSF-Bio tests, the water in both electrolyte chambers is significantly reduced in the anode chamber. The process of electrolysis of water at the anode $\left(2 \mathrm{H}_{2} \mathrm{O}\right.$ $\left.\rightarrow 4 \mathrm{e}^{-}+4 \mathrm{H}^{+}+\mathrm{O}_{2}\right)$ and in the cathode $\left(2 \mathrm{H}_{2} \mathrm{O}+2 \mathrm{e}^{-} \rightarrow\right.$ $2 \mathrm{OH}^{-}+\mathrm{H}_{2}$ ) and electroosmosis in the electrolyte chamber causes water volume reduction [4]. In contrast to PR-EKBio, in this test the water in both electrolyte chambers tends to be stable and does not disappear too much during electrokinetic process. This phenomena occurs because most of the electrolyzed ions re-form water (neutralization) when the polarity reversal occurs [4], [9]. 


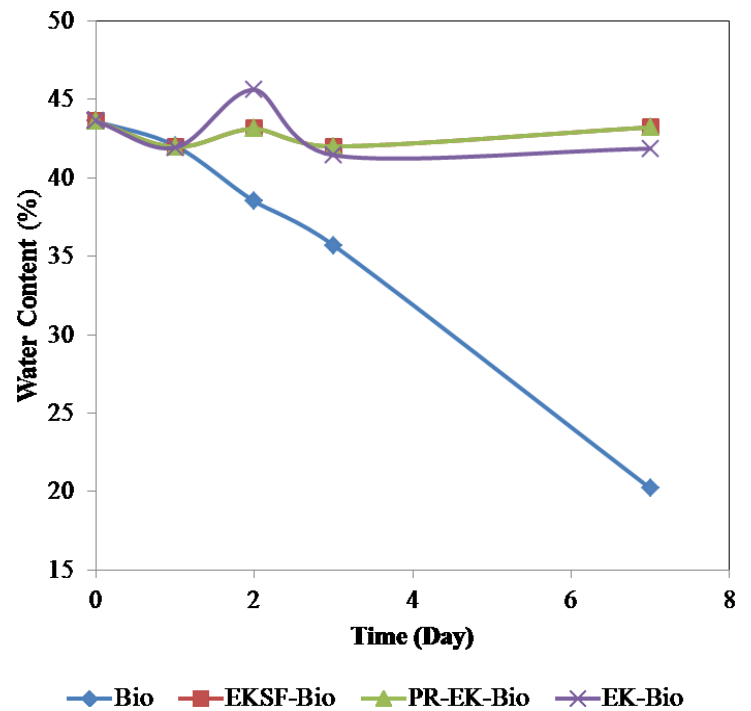

Fig. 3. Water content in soil profile

The distilled water may be replaced with mineral salt as both buffers and surfactants. Mineral salts may also serve as nutrients for microorganisms. Surfactant tween 80 used in this study serves to reduce the surface tension of oil droplet so it can solubilize in water and transported through electroosmosis mechanism. Surfactants are also capable of making micelle oil that can increase bioavailability and make oil suspended in soil-water pore and move through the electrophoresis process. PR-EKBio and EKSF-Bio have stable levels of moisture content when compared to EK-Bio so combination of surfactants with polarity reversals technique may provide favorable environmental conditions for electrokinetic bioremediation processes [5], [10].

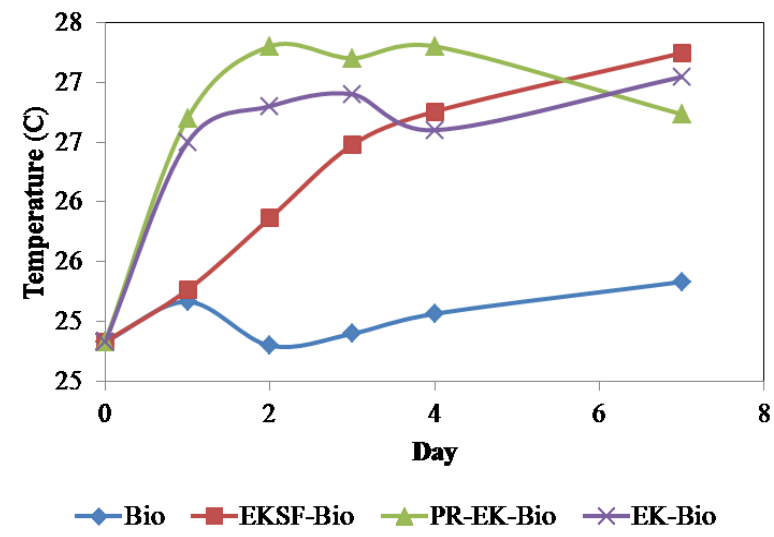

Fig. 4. Soil temperature profile

Bearing $\mathrm{pH}$ values to protect microorganisms from death is an important factor in this study. Acidity / alkalinity of the system is highly dependent to water hydrolysis and ion migration due to electro migration. The anode chamber will be filled with negative ions from the soil and produce an acidic condition whereas the cathode chamber is filled with positive ions and produces an alkaline condition. In this study, a very rapid $\mathrm{pH}$ change occurs where on the third day, the average $\mathrm{pH}$ drop to the range of 4-5. Overall, the average $\mathrm{pH}$ decreases toward acidic ambience. This limitation can be maintained by using electrolyte solution recirculation or polarity reversal technique. Changes in $\mathrm{pH}$ may affect microorganism consortium as it may alter some contaminant properties, catabolic pressure and also toxicity. However, the increasing of acidity will make soil bonds molecules release of hydrocarbon compounds easier [8], [13], [14]. Figure 5 shows the $\mathrm{pH}$ change over time.

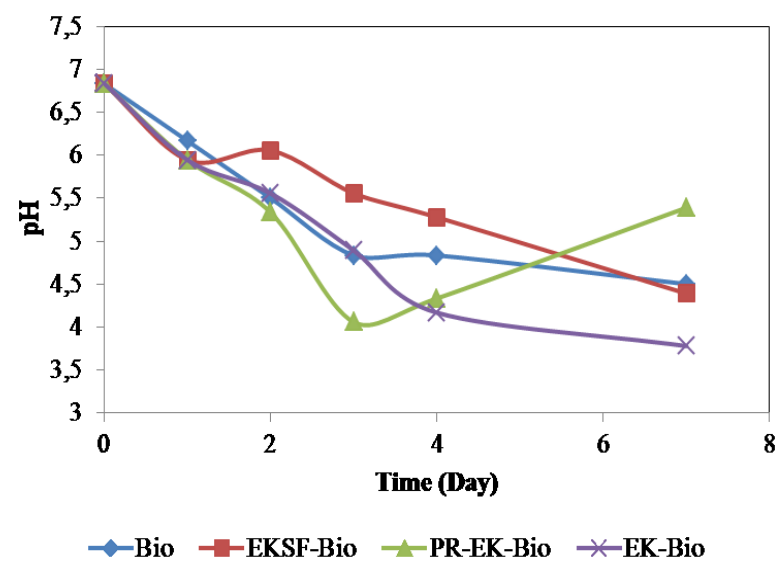

Fig. 5. Soil $\mathrm{pH}$ profile

\subsection{Microbial Distribution}

Electrokinetic process can improve bioremediation process because in addition to delivering nutrients, acceptor and electron donors and contaminants, this process is able to move the microorganisms through the pores of the low permeability soil. The movement velocity of microorganisms ranges from $0.05-0.15 \mathrm{~cm}^{2}$ / $\mathrm{Vh}$ and hydrophobic contaminants of $0.001-0.26 \mathrm{~cm}^{2} /$ Vh depending on their specific nature / substance. In the process of electrokinetic bioremediation, electroosmosis is the primary transport mechanism that carries microorganisms and contaminants through the soil pores. The major obstacles in the bioremediation process in clay soil can be overcome by using this transport mechanism [7].

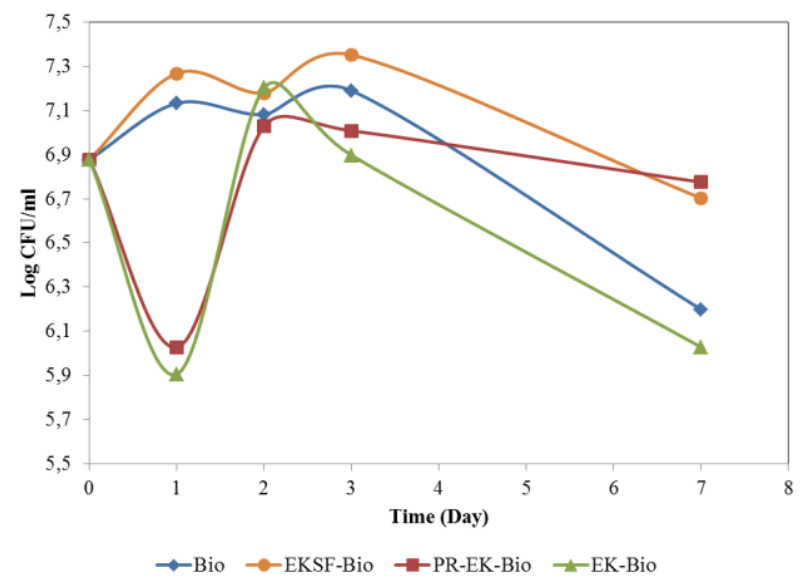

Fig. 6. Total microorganism in soil (log cfu/ml) 
Figure 6 shows that EKSF-Bio has the greatest number of microorganisms when compared with other tests. This relates to the surfactant's ability to make the oil dissolved in water. In addition, the surfactant may serve as a useful co-substrate for microorganisms. The microorganisms at the beginning of the study amounted to $8 \times 10^{6} \mathrm{CFU} / \mathrm{ml}$ and on average increased on the third day before finally decreasing until the seventh day. This decrease occurs due to changes in drastic environmental conditions such as decreased $\mathrm{pH}$ and moisture content.

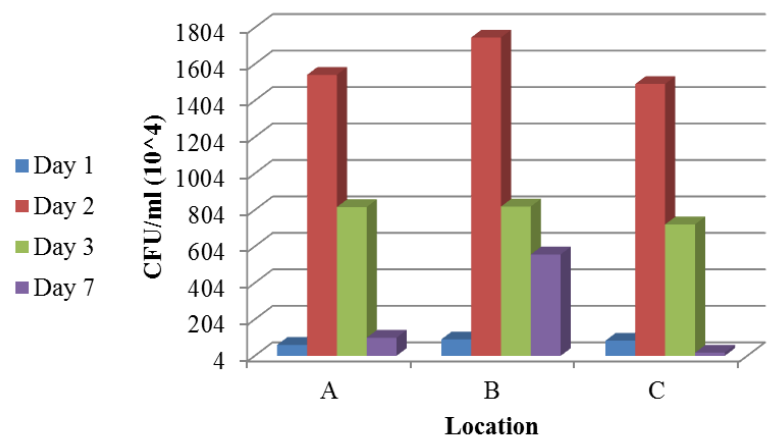

(a)

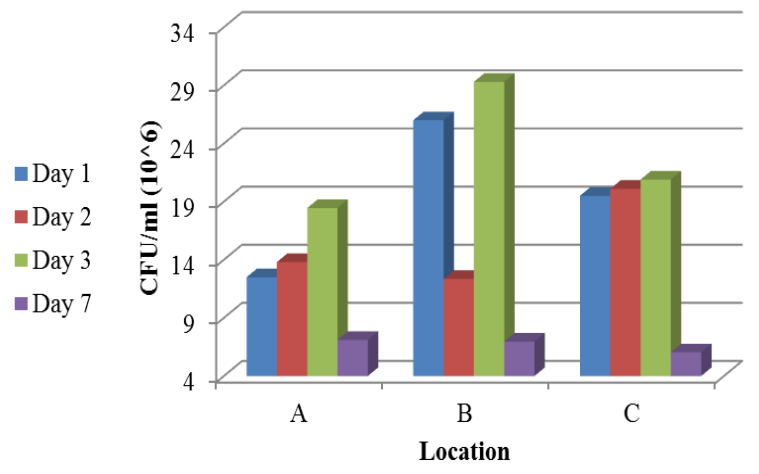

(b)

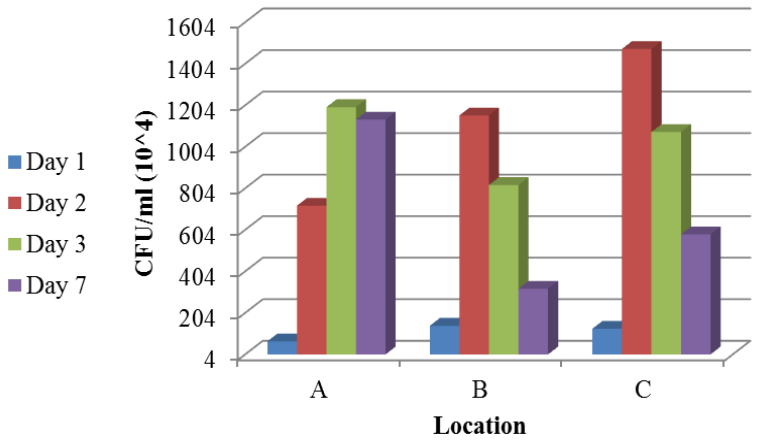

(c)

Fig. 7. Microorganism distribution under different test: a. EKBio; b. EKSF-Bio; c. PR-EK-Bio

The lowest number of microorganisms was found in the Bio test of $2 \times 10^{6} \mathrm{CFU} / \mathrm{ml}$. PR-EK-Bio can maintain the stability of the number of microorganisms in the range of $7 \times 10^{6}-1 \times 10^{7} \mathrm{CFU} / \mathrm{ml}$. The number of these microorganisms is greater when compared with other tests. This suggests the potential for the development of a combination of PR-EK-Bio and surfactant in subsequent studies. EK-Bio has the smallest number of microorganisms in the last day due to the high $\mathrm{pH}$ gap in the test reactor. In the anode, the $\mathrm{pH}$ value can reach 2 and 12 at the cathode. In contrast to EKSF-Bio is still able to maintain the $\mathrm{pH}$ between 3-11 and PR-EK-Bio of 4-10 at the end of the study.

The microorganisms near the cathode and anode chambers are lower than those located in the center of the reactor. In accordance with the results of the study shown in Fig. 7, microorganisms in the anode have smaller concentrations than cathodes. This relates to the acidic conditions near the anode chamber and the neutral conditions in the cathode chamber. This neutrality is due to much larger acid strength and almost affects the entire reactor. It also indicates the high negative ions contained in the soil. The distribution of microorganisms in polarity reversal tests is slightly different from the others due to transient effects on the system [12], [15].

\subsection{TPH Concentration}

Figure 8 shows the percentage of TPH in all four treatments conducted during the 7 days of the study. Table 2 shows that the volatile compounds are not left in the contaminated soil. The remaining hydrocarbon compounds are compounds with long carbon chains ranging from 15 to 21 . TPH removal in this study is assumed to be done by biological process and electrochemical phenomena since there is no further significant treatment of the process. The concentration of TPH fluctuates, this may occur as a result of contaminant transport factors at a particular point in the tested soil. Nevertheless, the downward trend is shown by all three electrokinetic tests.

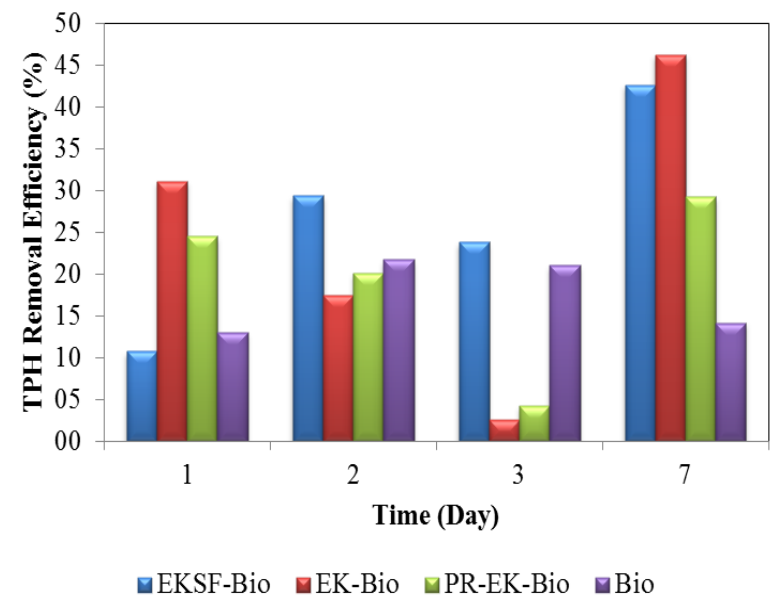

Fig. 8. TPH removal efficiency after 7 days run

The largest percentage of removal was in EK-Bio that was $46,3 \%$, followed by EKSF-Bio equal to $42,7 \%$ and PR-EK-Bio equal to $29,2 \%$. This condition is not in accordance with the original hypothesis which states that the reversal of polarity will be more effective when compared with ordinary electrokinetic processes. This can be happen because EKSF-Bio and EK-Bio have a lower $\mathrm{pH}$ value when compared to PR-EK-Bio. The $\mathrm{pH}$ value accelerates the process of solubilizing the hydrocarbon compounds into water so that the hydrocarbon 
compounds accumulate near the electrolyte chamber. In short, the electrolytes in both tests are rapidly depleted by evaporation and electrolysis processes. In bioremediation test (Bio), there is a decrease of TPH concentration although not as drastic using electrochemical process. This may be due to the mixing process and transport of microorganisms, soil and water at the beginning of the test causing contact between the active microorganisms and the contaminants so that the degradation process can occur [10], [14].

The percentage of degradation obtained is also not too large because of the very low in situ biodegradation. The TPH degradation is caused by two things, the transportation of contaminants due to the application of electrochemical and biological processes occurring in the soil. In EKSF-Bio, surfactants are able to mobilize TPH to microorganisms attached to the pores of the soil. At the end of the study, the surfactant was spread evenly on the soil and obtained an average degradation that was evenly distributed at each point. The electroosmosis process allows the presence of petroleum in the anode chamber. In this study, there is no research to know the TPH concentration in the electrolyte chamber. However, a larger TPH removal is likely to occur when prolonging the research process. Development carried out in this research can be an option in supporting the effectiveness of electrokinetic bioremediation process [16], [17].

\section{Conclusion}

The results show that in situ bioremediation process can be integrated either by electrokinetic process or by soil flushing. Polarity reversal technique is known to maintain the environmental conditions that are compatible with the growth of microorganisms. But there needs to be some improvement strategies to increase the degradation efficiency. Surfactants are known to improve the electrochemical process. A greater percentage of degradation is still possible if appropriate design process is found or extending the running time of the study. Electrokinetic bioremediation can be used to treat petroleum contaminated soil.

\section{Acknowledgement}

The authors acknowledge the financial support for this study from Environmental Engineering Department, ITB.

\section{References}

1. Q. Helmy, R. Laksmono, and E. Kardena, 2nd Humbold Kolleg in conjunction with International Conference on Natural Sciences, vol. 14, pp. 326-333, (2015)

2. T. Li et al., Electrochim. Acta, vol. 187, pp. 567-575, (2016).

3. D. Huang, Q. Xu, and J. Cheng, Int. J. Electrochem. Sci., vol. 7, pp. 4528-4544, (2012).

4. I. Hassan, E. Mohamedelhassan, E. K. Yanful, and Z. Yuan, Adv. Microbiol., vol. 6, no. January, pp. 57-72, (2016).

5. E. M. Ramírez, C. S. Jiménez, J. V. Camacho, M. A. R. Rodrigo, and P. Cañizares, Electrochim. Acta, vol. 181, pp. 192-199, (2015).

6. E. M. Ramirez, J. V. Camacho, M. A. Rodrigo, and P. Canizares, Sci. Total Environ., vol. 533, pp. 307-316, (2015).

7. R. T. Gill, M. J. Harbottle, J. W. N. Smith, and S. F. Thornton, Chemosphere, vol. 107, pp. 31-42, (2014).

8. L. Y. Wick et al., Sci. Total Environ., vol. 408, no. 20, pp. 4886-4893,(2010).

9. I. Hassan, E. Yanful, E. Mohamedelhassan, and M. W. Bo, "Enhanced electrokinetic bioremediation by $\mathrm{pH}$ stabilisation," (2016).

10. E. Mena, J. Villaseñor, P. Cañizares, and M. A. Rodrigo, Electrochim. Acta, vol. 190, pp. 294-304, (2016).

11. T. Li, S. Guo, L. Zhang, and F. Li, 2010 4th Int. Conf. Bioinforma. Biomed. Eng. iCBBE 2010, pp. 1-4, (2010).

12. Y. Yuan, S. Guo, F. Li, B. Wu, X. Yang, and X. Li, J. Hazard. Mater, vol. 320, pp. 591-601, (2016).

13. L. Y. Wick, P. A. Mattle, P. Wattiau, and H. Harms, Environ. Sci. Technol., vol. 38, no. 17, pp. 4596-4602, (2004).

14. O. H. P. and G. N. P. Adrián J. Acuña, New Technol. Oil Gas Ind., pp. 29-48, (2012).

15. F. Li, S. Guo, and N. Hartog, Electrochim. Acta, vol. 85, pp. 228-234, (2012).

16. T. Li, S. Guo, B. Wu, L. Zhang, and Y. Gao, J. Chem. Technol. Biotechnol., vol. 90, no. 3, pp. 441-448, (2015).

17. T. Li, S. Guo, B. Wu, F. Li, and Z. Niu, J. Environ. Sci, vol. 22, no. 9, pp. 1381-1386, (2010). 